\title{
Aplicación de un modelo de trazadores lagrangianos en Bahía Anegada, Argentina
}

Application of the Lagrangian tracers model in Anegada Bay, Argentina

\author{
Laura I. Alvarez ${ }^{1,2}$, Diana G. Cuadrado ${ }^{1,3}$ y Gerardo M. E. Perillo ${ }^{1,3}$ \\ ${ }^{1}$ Instituto Argentino de Oceanografía (IADO - CONICET), CC 804, B8000BFW, Bahía Blanca, Argentina. lalvarez@iado- \\ conicet.gob.ar \\ ${ }^{2}$ Departamento de Ingeniería, Universidad Nacional del Sur, Alem 1253, 8000 Bahía Blanca, Argentina \\ ${ }^{3}$ Universidad Nacional del Sur, Departamento de Geología, San Juan 670, 8000 Bahía Blanca, Argentina
}

\begin{abstract}
In this study we have applied a hydrodynamic numerical model of Lagrangian tracers to study the dispersion and subsequent settlement of particles from two different points of emission. The main objective was to define whether the particles, within the sedimentation period, could be exported out of the Anegada Bay (Argentina). We obtained the distance traveled by the particles, transported by currents induced by the tide and wind, and determined possible settlement areas. It was concluded that particles released from two different points do not leave Anegada Bay boundaries.
\end{abstract}

Key words: MOHID, hydrodynamic model, circulation

Resumen. - En el presente trabajo se ha realizado un experimento numérico utilizando un sistema de modelación hidrodinámico con trazadores lagrangianos para estudiar el transporte y circulación de partículas a partir de dos puntos de emisión. El objetivo fue establecer si las partículas pueden ser expulsadas de la Bahía Anegada (Argentina) dentro del plazo estimado para el período disponible antes de su sedimentación. Se obtuvo la distancia recorrida por las partículas, transportadas por las corrientes inducidas por la marea y el viento, y se determinó las posibles zonas de depósito. Se concluyó que las partículas emitidas en los dos puntos elegidos para este estudio permanecieron dentro de los límites de la Bahía Anegada.

Palabras clave: MOHID, modelo hidrodinámico, circulación

\section{INTRODUCCIÓN}

La implementación de modelos numéricos en zonas costeras se ha constituido en una herramienta necesaria para el diseño de planes de manejo costero. Su carácter predictivo permite tener un conocimiento cuantitativo y cualitativo de las diversas características físicas, biológicas y químicas de una región en particular.

Los modelos de transporte lagrangiano se utilizan para la modelación de eventos de derrame de petróleo (Shiau 1991), descargas cloacales (Cardini et al. 2004), modelos de circulación, etc. Estos modelos están basados en el concepto de trazador, cuya principal propiedad es su posición (x, y, z). Si se quiere estudiar el comportamiento de las corrientes entonces cada trazador estará relacionado con una masa de agua, en el caso de transporte de sedimento las partículas estarán relacionadas con la concentración de sedimento en suspensión. En el caso de los estudios biológicos los trazadores representarán celdas de zooplancton, fitoplancton o nutrientes.
Un fenómeno que puede ser simulado usando el esquema de trazadores lagrangianos es la invasión de especies en el hábitat costero, que ha sido un fenómeno frecuente en las últimas décadas. Como consecuencia de esta invasión, se produce un impacto significativo en el ecosistema receptor, disminuyendo la población de otras especies, generando conflictos en la disponibilidad de los alimentos (Ruesink et al. 2005).

Una de las especies invasoras más representativa de este fenómeno es la ostra Crassostrea gigas (Thumberg, 1793), también llamada ostra del Pacífico. Esta especie proviene de los mares de Japón, Corea y China y, actualmente, se le encuentra tanto en Europa como en América (See \& Feist 2009). Es la especie de ostra más cultivada en el mundo, correspondiendo al $80 \%$ del comercio internacional de ostras (Borges 2005). Los mayores productores son Francia, Japón y Corea. El éxito de su dispersión radica en la capacidad de adaptación al 
medio, tales como la adhesión de sus semillas, que pueden ejercer sobre cualquier superficie dura, superficies fangosas o arenosas o sobre otras especies de moluscos sésiles (Borges 2005). La ostra se caracteriza porque sus poblaciones se concentran en densas formaciones, excluyendo a otras especies nativas, provocando limitaciones en los alimentos y en la disponibilidad del espacio, lo que genera cambios sustanciales en el medio ambiente (Everett et al. 1995, Troost 2010).

La región de estudio, Bahía Anegada en Argentina presenta diferentes ambientes costeros, como marismas típicas, planicies de marea y playas de arena. Las planicies de abrasión por olas (restingas) se presentan discontinuas en algunos márgenes continentales (Dos Santos \& Borges 1995, Borges 1997). En dicha región Crassostrea gigas ha formado bancos mesolitorales en dos ambientes: en marismas bajas y en restingas de tosca, exhibiendo una importante expansión. En el año 2001 estos bancos tenían apenas 1 individuo $\mathrm{m}^{-2}$, mientras que al año siguiente (2002) la densidad fue de 40 individuo $\mathrm{m}^{-2}$ (Borges 2005).

El objetivo de este trabajo fue aplicar el modelo hidrodinámico MOHID, con su correspondiente módulo de trazadores lagrangianos, para caracterizar la circulación de agua en la región. En base a los trazadores lagrangianos se logró determinar las posibles zonas de influencia a partir de dos puntos de emisión particular, elegidos por ser los puntos de mayor concentración de ostras. El principal cuestionamiento fue establecer si las larvas de ostras emitidas en estos puntos pueden ser exportadas de la Bahía Anegada en 20 días, desde la eclosión hasta el asentamiento de las larvas. La aplicación de este modelo en el corto plazo, es un paso previo a la aplicación de un modelo que integre la biología de las larvas y antecedentes poblacionales.

\section{Materiales Y MÉtodos}

\section{ÁreA DE ESTUdio}

Bahía Anegada está situada al sur de la provincia de Buenos Aires, Argentina (Fig. 1) entre $39^{\circ} 45^{\prime}$ a $40^{\circ} 50^{\prime}$ 'S y $61^{\circ} 31^{\prime}$ a $62^{\circ} 52^{\prime} \mathrm{O}$, con un área estimada de $1400 \mathrm{~km}^{2}$. Esta zona es de escasa profundidad ( $5 \mathrm{~m}$ ) y el Este limita con el océano Atlántico con la presencia de varias islas (e.g., Gaviota, de los Riachos y Gama) en forma de barrera. Los canales que separan a las mencionadas islas son, de norte a sur: canal Culebra (14 m de profundidad), canal Oruga (10 m de profundidad) y canal San Blas (28 m de profundidad).

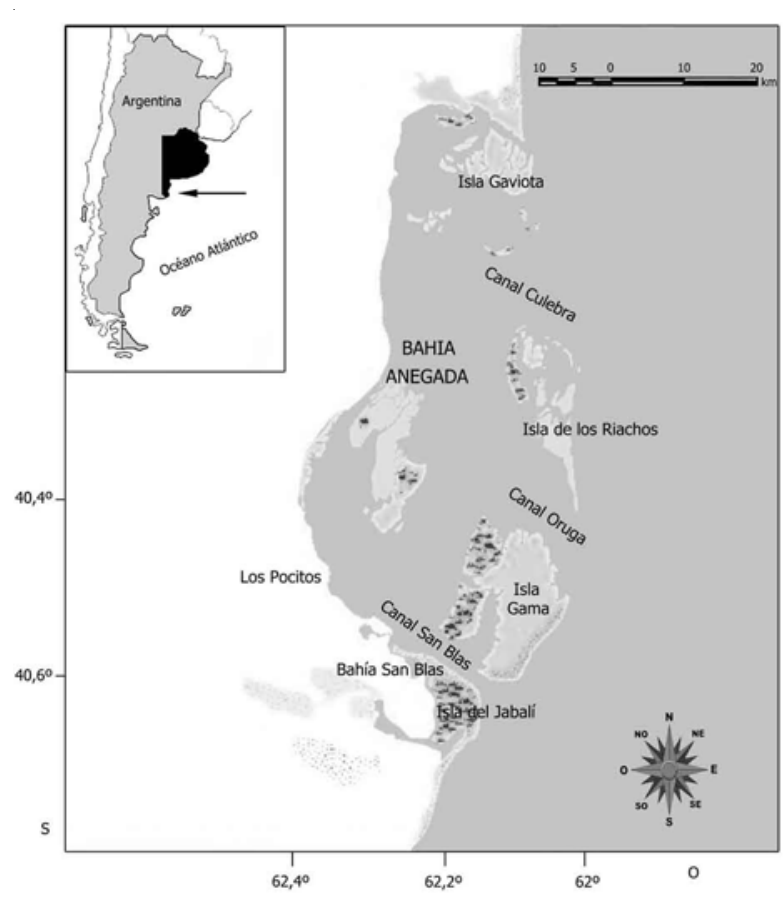

Figura 1. Área de estudio, Bahía Anegada / Study área, Anegada Bay

El sector específico de estudio involucra la zona sur de Bahía Anegada, entre Los Pocitos y el canal San Blas (Fig. 1). Este canal separa la denominada isla del Jabalí de la isla Gama y es el que posee la mayor profundidad. Corresponde a una entrada de marea, caracterizada por una geomorfología submarina que refleja un fondo plano carente de sedimentos no consolidados por el gran transporte de sedimentos como resultado de las fuertes velocidades de corrientes de marea medidas en la zona (Cuadrado \& Gómez 2010).

En esta región las temperaturas atmosféricas medias fluctúan de $1,6^{\circ} \mathrm{C}$ en invierno (junio, julio y agosto) y $22^{\circ} \mathrm{C}$ en verano (diciembre, enero y febrero), con una temperatura media anual de $11^{\circ} \mathrm{C}$. La temperatura oscila entre $9^{\circ} \mathrm{C}$ en invierno y $20^{\circ} \mathrm{C}$ en verano, mientras que la salinidad alcanza el valor mínimo de 26 en junio y máximo de 33 en septiembre, medidos sobre el veril sur entre Los Pocitos y bahía San Blas, la variación en la salinidad se debe a las precipitaciones estacionales, dado que el aporte de agua dulce continental es sumamente reducido (Borges 2005). En sentido vertical no hay variación de la salinidad, evidenciando el característico estado homohalino de zonas de gran energía por corrientes de marea. Las mareas son del tipo mixto o de desigualdades diurnas. Su amplitud es de 1,65 m para sicigias y de $1,57 \mathrm{~m}$ para cuadraturas (SHN 
2008) En las distintas simulaciones se utilizaron los datos meteorológicos de verano. En el experimento numérico solo se consideraron los datos de salinidad, temperatura y viento de los meses de verano, puesto que el objetivo fue simular sólo esa época del año y su relación con la emisión de las larvas de Crassostrea gigas.

\section{MoDELO HIDRODINÁMico}

Para modelar la circulación de agua en Bahía Anegada se utilizó el modelo hidrodinámico MOHID (Coelho et al. 2002, Santos et al. 2002), el cual está basado en el método de volúmenes finitos (Martins et al. 2001). Dicho modelo fue desarrollado por el grupo MARETEC del Instituto Técnico de Lisboa, Portugal.

El modelo plantea y resuelve las ecuaciones de cantidad de movimiento 3D en forma cartesiana asumiendo la validez de la aproximación de Boussinesq. Las tres componentes de la velocidad fueron tratadas usando una grilla del tipo Arakawa-C (Arakawa \& Lamb 1977). Básicamente, consta de diferentes módulos mediante los cuales se pueden integrar datos hidrodinámicos, geomorfológicos, meteorológicos, biológicos, etc. Utiliza un algoritmo semi-implícito ADI (Alternating-Direction Implicit) para la resolución de la ecuación de advección-difusión, con una reducción importante en los costos computacionales. Este método numérico semi-implícito se basa en la división del paso temporal en dos etapas $(t \rightarrow t+1 / 2$ y t $+1 / 2 \rightarrow t+1)$ donde, en cada medio paso de tiempo, se resuelve en forma implícita una dimensión por vez (Fig. 2), en la figura se observa que en la primera etapa de tiempo de integración $(t \rightarrow t+1 / 2)$ la dirección ' $y$ ' se trabaja en forma implícita, mientras que en la dirección ' $x$ ' en forma explícita, en la segunda etapa ( $t+1 / 2 \rightarrow t+1)$ ocurre lo contrario, la dirección ' $x$ ' se trabaja en forma implícita mientras que la dirección 'y’ se trabaja en forma explícita.

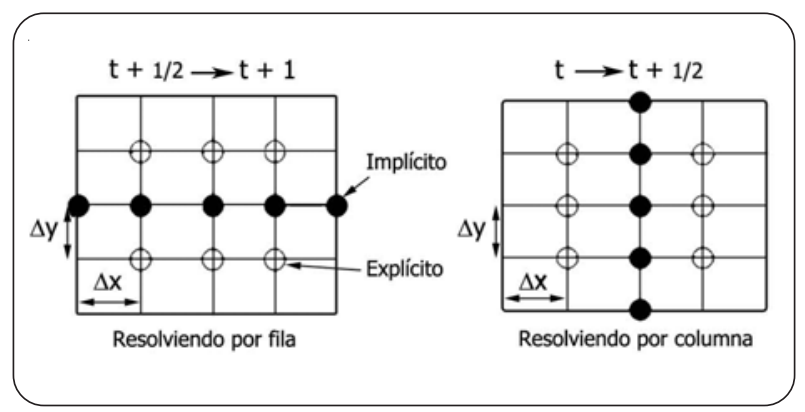

Figura 2. Esquema ilustrativo del algoritmo semi-implícito ADI / Schematic illustration of the semi-implicit ADI algorithm
Para la dirección horizontal se utilizaron coordenadas cartesianas mientras que en sentido vertical se usaron coordenadas del tipo Sigma. MOHID tiene la opción de usar varios tipos de clausura turbulenta. Está acoplado con el modelo General Ocean Turbulence Model (GOTM) (Burchard et al. 1999), que trata en forma numérica los procesos de mezcla vertical dentro de la columna de agua, entre otros aspectos. Para este estudio fue configurado en el esquema k-e para el cálculo de los coeficientes de viscosidad y difusividad turbulentos (Burchard \& Bolding 2001).

El modelo implementado fue constituido por dos modelos anidados con diferente resolución. El primer nivel cubre la costa del Atlántico Sur $\left(70^{\circ}-30^{\circ} \mathrm{O}, 60^{\circ}-20^{\circ} \mathrm{S}\right)$ con una resolución de $0,05^{\circ}$, con datos provenientes del atlas digital GEBCO ${ }^{1}$, base de datos globales batimétricos, con una resolución global de un minuto. El segundo nivel cubre el sector de Bahía Anegada $\left(62,5^{\circ}-61,6^{\circ} \mathrm{O}, 40,70^{\circ}\right.$ $38,8^{\circ} \mathrm{S}$ ) con una resolución de $0,004^{\circ}$. Para este nivel se digitalizó la información proveniente de la carta náutica disponible del Servicio de Hidrografía Naval (SHN 1999) y mediciones propias del canal San Blas. Luego, se generó la grilla rectangular numérica de batimetría por medio del programa MOHID GIS.

Como forzantes del modelo de mayor extensión se utilizaron datos meteorológicos medios estacionales de la base de datos de la $\mathrm{NOOA}^{2}$, en particular datos de dirección y magnitud de viento, temperatura superficial y datos de marea extraídos del modelo global Fes2004, que corresponde a una versión actualizada del Fes95.2 (Le Provost et al. 1998). El estado inicial del modelo fue de velocidad y nivel de agua nulos, por lo cual fue necesario simular el comportamiento del sistema durante un período de 20 días para lograr su estabilización. Luego del mismo se simularon 90 días, correspondientes al período diciembre-marzo. En la frontera se utilizó el esquema de radiación de Blumberg y Kantha (Blumberg \& Kantha 1984).

El modelo de menor extensión fue forzado por la marea heredada del modelo de mayor extensión y por la serie de tiempo registrada por la estación EMAC (Estación de Monitoreo Ambiental Costero), perteneciente al Instituto Argentino de Oceanografía (IADO) $\left(62,25^{\circ} \mathrm{O} ; 40,54^{\circ} \mathrm{S}\right)$, referida a la dirección y magnitud del viento, dado que los vientos globales poseen resolución insuficiente para

\footnotetext{
${ }^{1} \measuredangle$ ttp:// www.ngdc.noaa.gov/ mgg/gebco/gebco. html> ${ }^{2} \triangleleft$ http:// www.esrl. noaa.gov/ psd/ data/ index.html>
} 
la escala de trabajo. Se utilizó el esquema de radiación de Blumberg y Kantha (Blumberg \& Kantha 1984) para las fronteras laterales y el esquema de fronteras móviles para la costa, con el objeto de representar la situación de cubrimiento y descubrimiento de ciertas zonas del área a modelar.

\section{Modelo de TRAZAdores LAgRANGianos}

Los modelos de dispersión lagrangianos utilizan el concepto de Trazadores (modelo de rastreo de partículas). La principal característica de estos modelos es evitar la resolución explícita del término advectivo de la ecuación de transporte, para evadir el problema de la inestabilidad. La precisión resulta del número de trazadores e indirectamente del campo de velocidades y de los parámetros característicos de turbulencia local (viscosidad turbulenta, longitud de mezclado y varianza de la velocidad turbulenta). Se utilizó para la advección un esquema mixto upwind y diferencias centrales.

En el presente estudio, se utilizó el MOHID con su correspondiente módulo de trazadores lagrangiano en dos simulaciones, para representar en forma cualitativa la dispersión de partículas, la primera con foco de emisión sobre la costa a nivel del mar, en Los Pocitos y la segunda con foco de emisión en cercanías de bahía San Blas (Fig. 1). La hidrodinámica utilizada en los cálculos de la posición de los trazadores proviene del modelo hidrodinámico estabilizado. Se emitieron 10 partículas por hora durante los primeros 15 días de la simulación de 45 días. Las partículas se encuentran en la simulación durante un lapso de tiempo de 20 días, tomados desde el momento de la emisión, en concordancia con una posible aplicación de la invasión de Crassostrea gigas, según al tiempo de vida larval de la ostra. Durante esos 20 días las partículas pueden estar en suspensión en el agua, sobre la costa, o bien transcurrido este período de tiempo, el modelo las elimina.

El objeto de la elección de dichos puntos y tiempo de emisión se debe a la alta concentración de ostras Crassostrea gigas, tanto en la zona de bahía San Blas como en Los Pocitos. La densidad media de ostras en la

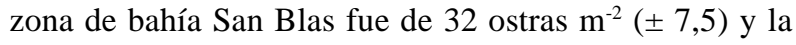
máxima concentración fue de 163 ostras $\mathrm{m}^{-2}(\mathrm{~N}=40)$ (Borges 2002) y en la zona de Los Pocitos la densidad promedio de ostras fue de 34 ostras $\mathrm{m}^{-2}( \pm 5)(\mathrm{N}=102)$, con una concentración máxima de 300 ostras $\mathrm{m}^{-2}$ (Borges et al. 2001).

\section{Resultados}

\section{HidRodinÁMiCA dE LA REgión dE BAHÍA ANEGADA}

En el análisis de los datos provenientes de la estación EMAC se obtuvo que la dirección de viento de mayor frecuencia fue la proveniente del sector N-NNE (Fig. 3a), con un promedio anual de $35 \mathrm{~km} \mathrm{~h}^{-1}$ (Fig. 3b). Los vientos variaron de acuerdo a la estación del año, siendo la primavera y el verano las estaciones más ventosas, con una velocidad promedio de $30,6 \mathrm{~km} \mathrm{~h}^{-1}$ y 33,7 $\mathrm{km} \mathrm{h}^{-1}$, respectivamente.
Figura 3. Análisis de los datos de viento de la zona de estudio. A) Frecuencia de ocurrencia. B) Velocidad del viento promedio / Wind data analysis obtained in the study zone. A) Frequency of occurrence. B) Average wind velocity
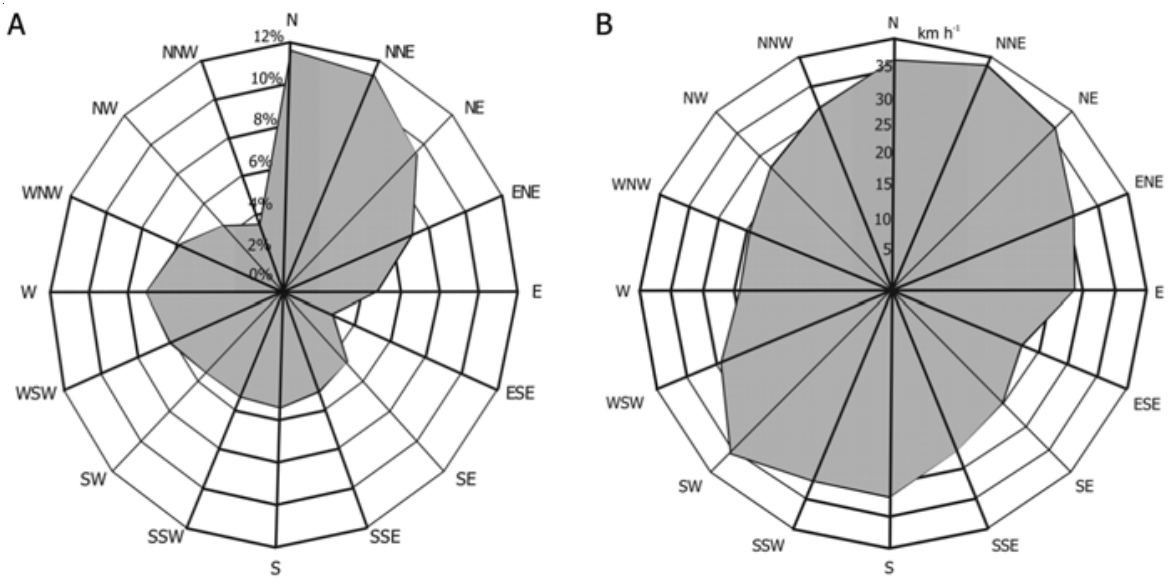
Los resultados obtenidos del software de postprocesado del MOHID con referencia al modelo hidrodinámico, mostraron que las máximas velocidades se registraron en el canal San Blas, alcanzando 1,85 $\mathrm{m} \mathrm{s}^{-1}$ durante el reflujo y, 1,97 durante el flujo, estos valores concuerdan con mediciones realizadas (Cuadrado \& Gómez 2010). En el resto de Bahía Anegada las velocidades de corrientes de marea resultaron inferiores a $1 \mathrm{~m} \mathrm{~s}^{-1}$, excepto en el canal Culebra con un rango de variación de entre 0,8 y $1,6 \mathrm{~m} \mathrm{~s}^{-1}$. Durante el estado de marea creciente la magnitud de la velocidad de ingreso de agua a la bahía es similar en tres canales, de norte a sur: canales Oruga, Culebra y San Blas (Fig. 4a), mientras que durante el estado de marea bajante la magnitud de la velocidad es mayor en el canal San Blas (Fig. 4b).

Para validar el modelo hidrodinámico se compararon los datos de nivel de marea registrados por la estación EMAC con los calculados por el modelo, de esta manera se reconoce una correspondencia entre las dos series de tiempo entre la marea media y la simulada con similar patrón de variación.

\section{DistribuCión LAGRANGIANA}

Los resultados obtenidos postprocesado del MOHID en relación al transporte de partículas, estuvieron directamente relacionados con los resultados de la hidrodinámica de la región. El resultado de la primera simulación mostró que las partículas con frente de emisión en Los Pocitos durante el flujo tienen un desplazamiento en la dirección $\mathrm{N}$ de 15,1km, en dirección NE de 15,8km
(Fig. 5a) y durante el reflujo la dirección predominante fue SE, con una distancia de 10,9 km (Fig. 5b).

En el segundo experimento se consideró que el frente de emisión se encuentra entre Los Pocitos y la bahía San Blas $\left(62,183^{\circ} \mathrm{O}-40,577^{\circ} \mathrm{S}\right)$. El resultado de esta simulación mostró una distribución con las mismas características a la anterior. Las direcciones de transporte coincidieron con las direcciones de las corrientes. Sin embargo, las partículas que fueron desplazadas en la dirección NE llegaron a la isla Gama (Fig. 5c), recorriendo una distancia de aproximadamente $15 \mathrm{~km}$. Las otras dos direcciones de las partículas fueron de 8,5 km hacia el NO, en dirección a Los Pocitos y 11,3 km en dirección SE sobre la costa sur del canal San Blas (Fig. 5d).

\section{Discusión}

El modelo MOHID ha sido muy utilizado en estuarios (Martins et al. 2001), áreas costeras (Villarreal et al. 2002) y océano abierto (Santos et al. 2002) entre otros, con resultados satisfactorios. En el presente trabajo, el modelo tuvo en cuenta la influencia del viento promedio estacional y la marea como únicos forzantes, bajo el supuesto de que el viento es una gran influencia en la circulación de regiones de escasa profundidad (Levasseur et al. 2007). Con estas condiciones se determinó en forma cualitativa que la hidrodinámica de la zona de Bahía Anegada se encuentra fuertemente influenciada por la geomorfología. Durante el flujo, el agua ingresa a la bahía por cuatro canales, con velocidades de magnitud similar en todos los casos, mientras que durante el reflujo, la exportación
Figura 4. Resultados del modelo Hidrodinámico. A) Marea de flujo. Entrada de agua a la bahía a través de varios canales. B) Marea de reflujo. Salida de la masa de agua a través del canal San Blas principalmente / Hydrodynamic model output. A) Tidal flood. The entry of water into the bay through the channels. B) Tidal ebb occurring mainly through San Blas Channel
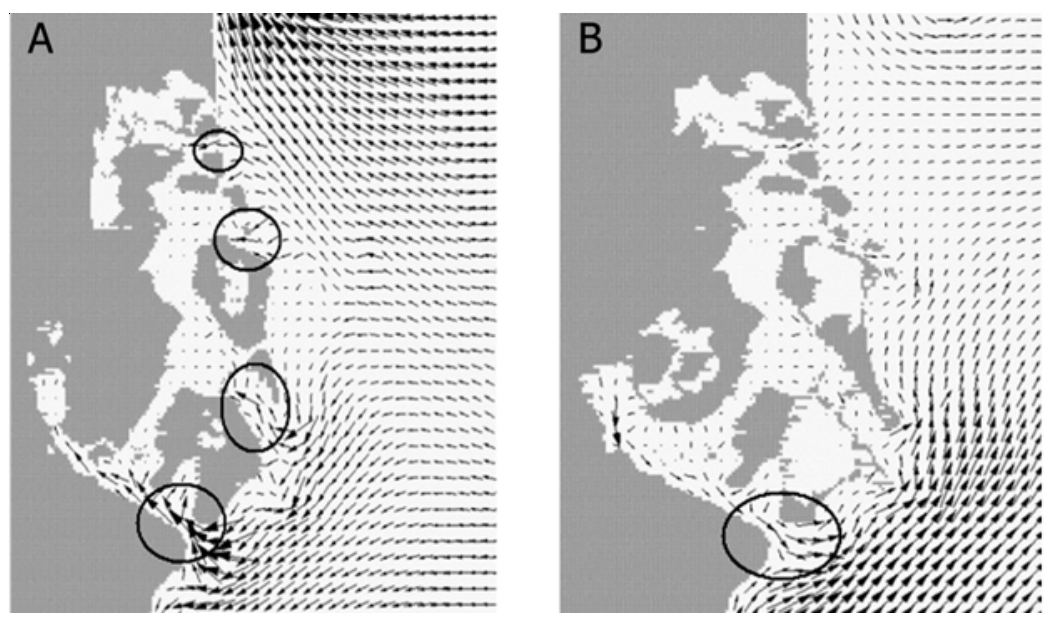
de agua se produce con mayores velocidades por el canal San Blas. Estos resultados, al igual que los obtenidos por medio de la aplicación del modelo MOHID en los Fiordos Australes de Chile (Marín \& Campuzano 2006) demuestran que la aplicación del modelo reproduce eficientemente la dinámica en diferentes regiones de estudio.

El modelo numérico de trazadores es una herramienta adecuada para estudiar la circulación de partículas. Su utilización ha permitido determinar posibles zonas de depósito de partículas a partir de dos puntos de emisión. La dispersión de las partículas con frente de emisión en
Los Pocitos ocurrió en todas las direcciones alcanzando una distancia máxima de $19 \mathrm{~km}$ hacia el interior de la bahía y mínima de 10 km, en dirección SE. La dispersión de las partículas que tienen como frente de emisión el norte de bahía San Blas logró alcanzar la isla Gama, ubicada en la costa opuesta del canal. Por otro lado, el depósito de partículas alcanzó una distancia máxima de 11 km sobre la costa sur del canal. Por su parte, otros autores estiman que el rango de distancia máxima recorrida por partículas, en este caso la ostra Crassostrea gigas durante su vida planctónica, fue de $50 \mathrm{~km}$ (Shanks et al. 2003, Siegel et al. 2003), debido a las diferencias hidrodinámicas de cada región.

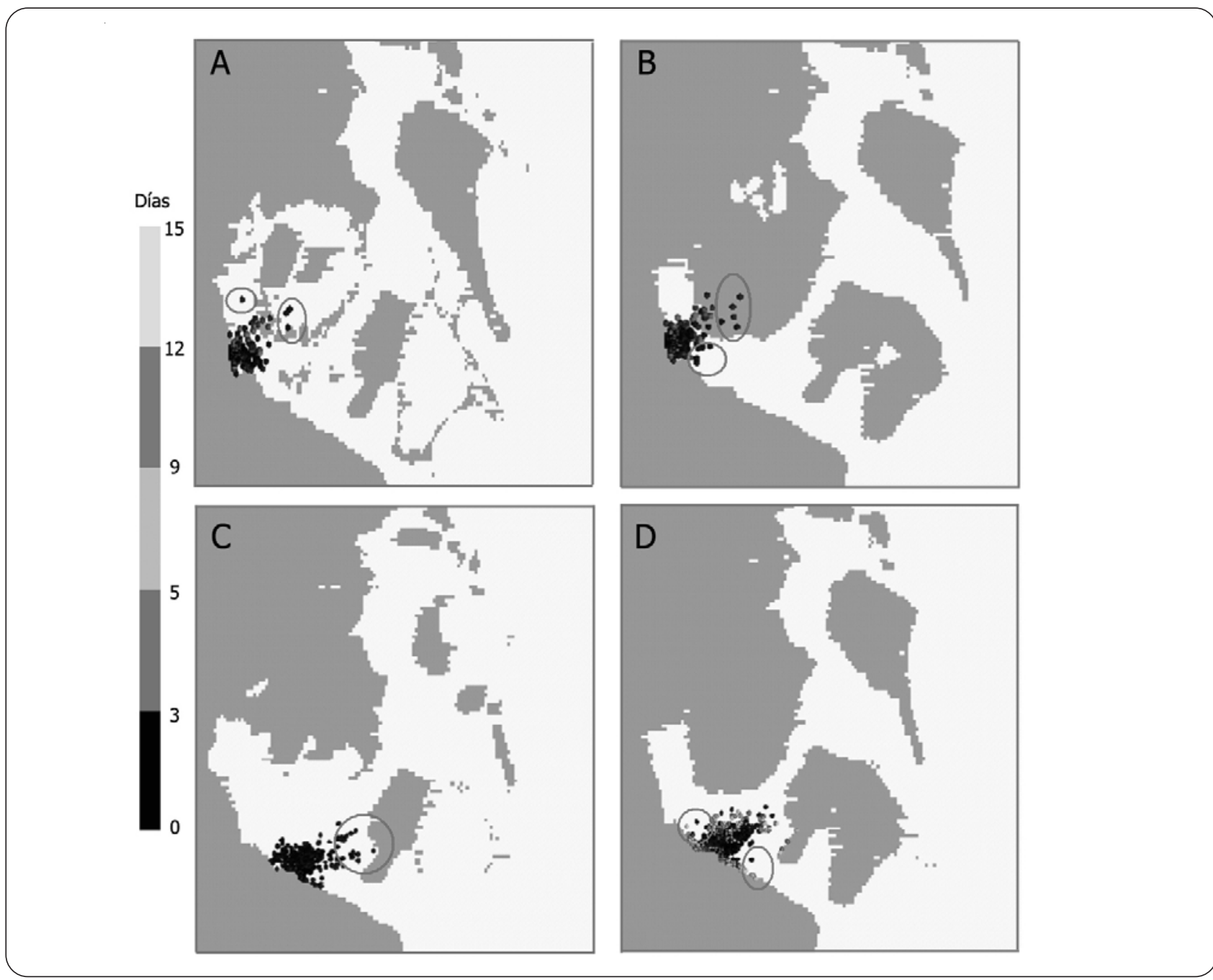

Figura 5. Resultados del modelo de trazadores Lagrangianos. A) Desplazamiento de las partículas con punto de emisión en Los Pocitos durante la marea de flujo. B) Desplazamiento de las partículas durante la marea de reflujo con punto de emisión en Los Pocitos. C) Desplazamiento de las partículas con punto de emisión en San Blas durante la marea de flujo. D) Desplazamiento de las partículas con punto de emisión en San Blas durante la marea de reflujo / Results of Lagrangian tracers model. A) Displacement of particles during tidal flood with emission point in Los Pocitos. B) Displacement of particles during the ebb tide with the emission point in Los Pocitos. C) Emission point in San Blas during flood tide. D) Displacement of particles with emission point in San Blas during ebb tide 
Por lo expuesto, se puede inferir que las partículas, con los frentes de emisión antes mencionados, se diseminan dentro de Bahía Anegada sin superar los 20 km de distancia desde el punto de emisión. En ninguno de los casos las emisiones permitieron que las partículas abandonen la Bahía Anegada y sean exportadas hacia el océano Atlántico. Futuras simulaciones permitirán determinar la potencial localización de los bancos de ostras como puntos emisores que favorecen la exportación de larvas hacia el exterior de Bahía Anegada con el fin de determinar estrategias de control y establecer como ha sido la evolución de los asentamientos desde la primera colonización.

\section{Agradecimientos}

Los autores agradecen al Sr. Ernesto Alberdi las oportunas sugerencias y los datos aportados y a la Dra. Clara Menéndez por sus atinadas correcciones y comentarios. Asimismo agradecen los aportes de dos revisores anónimos y del editor de la revista que enriquecieron significativamente el manuscrito. Este trabajo fue financiado por PAE No22666, financiado por la ANPCyT y PGI 24/ZH15 perteneciente a SCyT-UNS

\section{LITERATURA CITADA}

Arakawa A \& V Lamb. 1977. Computational design of the basic dynamical processes of the UCLA general circulation model. En: Chang J (ed). Methods in computational physics 17: 174-267. Academic Press, New York.

Blumberg AF \& LH Kantha. 1984. Open boundary condition for circulation models. Journal of Hydraulic Engineering 111(2): 237-255.

Borges ME. 1997. Conservación de la Bahía Anegada. Informe 17/97: 1-15. Ministerio de Asuntos Agrarios, Dirección Provincial de Pesca, La Plata.

Borges ME. 2002. Ubicación y biomasa de los bancos de ostras en Bahía Anegada. Informe Técnico 1: 1-19. Ministerio de Agricultura, Ganadería y Pesca de la Provincia de Buenos Aires, Subsecretaría de Actividades Pesqueras, La Plata.

Borges ME. 2005. La ostra del Pacífico, Crassostrea gigas (Thumberg, 1793) en la Bahía Anegada (Provincia de Buenos Aires). En: Penchaszadeh PE (ed). Invasores, pp. 310-356. Eudeba, Buenos Aires.

Borges ME, N Cazzaniga \& EP Dos Santos. 2001. Density and growth of Pacific oysters, Crassostrea gigas, in an intertidal oyster-reef in the southern Atlantic Coast of Buenos Aires province, Argentina. IB03-46, Mar del Plata. [CD-ROM]
Burchard H \& K Bolding. 2001. Comparative analysis of four second-moment turbulence closure models for the oceanic mixed layer. Journal of Physical Oceanography 31: 1943-1968.

Burchard H, K Bolding \& MR Villarreal. 1999. GOTM, a general ocean turbulence model: Theory, implementation and test cases. European Commission, Report EUR 18745: 1-103. [en línea] <http://www.io-warnemuende.de/tl_files/ staff/burchard/pdf/papers/report.pdf>

Cardini J, A Zabalett, N Oliver \& D Mársico. 2004. Efecto de los coeficientes de dispersión sobre la modelación bidimensional del transporte de contaminantes. Caso de estudio. Mecánica Computacional, Bariloche 23: 1201-1215.

Coelho HS, RJ Neves, M White, PC Leitão \& AJ Santos. 2002. A model for ocean circulation on the Iberian coast. Journal of Marine Systems 32: 153-179.

Cuadrado DG \& EA Gómez. 2010. Geomorfología y dinámica del canal San Blas, provincia de Buenos Aires (Argentina). Latin American Journal of Sedimentology and Basin Analysis 17(2): 1-22.

Dos Santos EP \& ME Borges. 1995. Contribución al conocimiento de la Bahía Anegada, partido de Patagones, prov. Buenos Aires, 70 pp. COLACMAR, Mar del Plata.

Everett RA, GM Ruiz \& JT Carlton. 1995. Effect of oyster mariculture on submerged aquatic vegetation: an experimental test in a Pacific Northwest. Marine Ecology Progress Series 125: 205-217.

Le Provost C, F Lyard, JM Molines, ML Genco \& F Rabilloud. 1998. A hydrodynamic ocean tide model improved by assimilating a satellite altimeter derived data set. Journal of Geophysical Research 103: 5513-5529.

Levasseur A, L Shi, NC Wells, DA Purdie \& BA KellyGerreyn. 2007. A three-dimensional hydrodynamic model of estuarine circulation with an application to Southampton Water, UK. Estuarine, Coastal and Shelf Science 73: 753767.

Marín VH \& FJ Campuzano. 2006. Un modelo hidrodinámicobarotrópico para los fiordos australes de Chile entre los $41^{\circ} \mathrm{S}$ y los $46^{\circ} \mathrm{S}$. Ciencia y Tecnología del Mar 31(2): $125-$ 136. [en línea] < http://www.cona.cl/ctmol2/vol31-2/ 07\%20marin.pdf $>$.

Martins F, PC Leitão, A Silva \& R Neves. 2001. 3D modelling of the Sado Estuary using a new generic vertical discretization approach. Oceanologica Acta 24(1): 51-62.

Ruesink JL, HS Lenihan, AC Trimble, KW Heiman, F Micheli, JE Byers \& M Kay. 2005. Introduction of nonnative oysters: ecosystem effects and restoration implications. Annual Review of Ecology, Evolution and Systematic 36: 643-689. 
Santos A, H Martins, H Coelho, PC Leitao \& R Neves. 2002. A circulation model for the European ocean margin. Applied Mathematical Modelling 26: 563-582.

See KE \& BE Feist. 2009. Reconstructing the range expansion and subsequent invasion of Introduced European green crab along the west coast of the United States. Biological Invasions 12: 1305-1318.

SHN. 1999. Carta Náutica, De Faro El Rincón a Faro Segunda Barranca. H213. Servicio de Hidrografía Naval, Buenos Aires.

SHN. 2008. Tablas de marea, H610, pp. 195-199. Servicio de Hidrografía Naval, Buenos Aires.

Shanks AL, BA Grantham \& MH Carr. 2003. Propagule dispersal distance and the size and spacing of marine reserves. Ecological Application 13(1): 159-169.
Shiau B. 1991. Computer modelling of oil pollutants transport on the water. Computer Modelling in Ocean Engineering 91: 467-475.

Siegel DA, BP Kinlan, B Gaylord \& SD Gaines. 2003. Lagrangian descriptions of marine larval dispersion. Marine Ecology Progress Series 260: 83-96.

Troost K. 2010. Causes and effects of a highly successful marine invasion: Case-study of the introduced Pacific oyster Crassostrea gigas in continental NW European estuaries. Journal of Sea Research 64: 145-165, <doi:10.1016/ j.seares.2010.02.004>

Villarreal MR, P Montero, JJ Taboada, R Prego, PC Leitão \& V Pérez-Villar. 2002. Hydrodynamic model study of the Ria de Pontevedra under estuarine conditions. Estuarine, Coastal and Shelf Science 54: 101-113.

Recibido el 14 de diciembre de 2010 y aceptado el 30 de mayo de 2011 